\title{
Oral Zinc Supplementation for the Treatment of Acute Diarrhea in Children: A Systematic Review and Meta-Analysis
}

\author{
Laura M. Lamberti ${ }^{1}$, Christa L. Fischer Walker ${ }^{1, *}$, Kit Y. Chan ${ }^{2,3}$, Wei-Yan Jian ${ }^{2}$ \\ and Robert E. Black ${ }^{1}$
}

1 Department of International Health, Johns Hopkins Bloomberg School of Public Health, 615 N. Wolfe St, Baltimore, MD 21205, USA; E-Mails: 1lambert@jhsph.edu (L.M.L.); rblack@jhsph.edu (R.E.B.)

2 Department of Health Policy and Management, School of Public Health, Peking University Health Science Centre, 38 Xueyuan Rd. in Haidian District, Beijing 10083, China; E-Mails:k.chan@ed.ac.uk (K.Y.C.); jianweiyan@bjmu.edu.cn (W.-Y.J.)

3 Centre for Population Health Sciences, University of Edinburgh Medical School, Teviot Place, Edinburgh, Scotland EH8 9AG, UK

* Author to whom correspondence should be addressed; E-Mail: cfischer@jhsph.edu; Tel.: +1-410-502-3478; Fax: +1-410-955-7159.

Received: 4 September 2013; in revised form: 9 October 2013 / Accepted: 4 November 2013 / Published: 21 November 2013

\begin{abstract}
Evidence supporting the impact of therapeutic zinc supplementation on the duration and severity of diarrhea among children under five is largely derived from studies conducted in South Asia. China experiences a substantial portion of the global burden of diarrhea, but the impact of zinc treatment among children under five has not been well documented by previously published systematic reviews on the topic. We therefore conducted a systematic literature review, which included an exhaustive search of the Chinese literature, in an effort to update previously published estimates of the effect of therapeutic zinc. We conducted systematic literature searches in various databases, including the China National Knowledge Infrastructure (CNKI), and abstracted relevant data from studies meeting our inclusion and exclusion criteria. We used STATA 12.0 to pool select outcomes and to generate estimates of percentage difference and relative risk comparing outcomes between zinc and control groups. We identified 89 Chinese and 15 non-Chinese studies for the review, including studies in 10 countries from all WHO geographic regions, and analyzed a total of 18,822 diarrhea cases (9469 zinc and 9353 control). None of the included Chinese studies had previously been included in
\end{abstract}


published pooled effect estimates. Chinese and non-Chinese studies reported the effect of therapeutic zinc supplementation on decreased episode duration, stool output, stool frequency, hospitalization duration and proportion of episodes lasting beyond three and seven days. Pooling Chinese and non-Chinese studies yielded an overall 26\% (95\% CI: $20 \%-32 \%$ ) reduction in the estimated relative risk of diarrhea lasting beyond three days among zinc-treated children. Studies conducted in and outside China report reductions in morbidity as a result of oral therapeutic zinc supplementation for acute diarrhea among children under five years of age. The WHO recommendation for zinc treatment of diarrhea episodes should be supported in all low- and middle-income countries.

Keywords: zinc; children; global health; China

\section{Introduction}

In response to mounting evidence supporting the efficacy and effectiveness of therapeutic zinc supplementation for diarrhea among children under five years of age, the World Health Organization (WHO) and the United Nation's Children Fund (UNICEF) issued a global recommendation in 2004, which advised zinc supplementation in addition to oral rehydration solution (ORS) for the treatment of all diarrhea episodes among children $<5$ years of age [1,2]. Systematic reviews have quantified the association between therapeutic zinc supplementation and a reduction in the duration and severity of childhood diarrhea episodes in low- and middle-income countries (LMICs) [1,3,4]. Many of the studies contributing to this body of evidence were conducted in South Asia [5-7], but literature stemming from East Asia has not been included in past reviews. In 2011, Zhang published a systematic review which identified 11 Chinese studies assessing zinc treatment for diarrhea and signified the need to update previous meta-analyses with literature published in languages other than English [8].

We sought to conduct an extensive search for studies of oral therapeutic zinc supplementation published in Chinese and any other language. We also aimed to combine evidence across regions in order to generate global estimates of the effect of oral therapeutic zinc supplementation on selected morbidity and mortality outcomes among children under five years of age.

\section{Methods}

We conducted a systematic literature search for studies published in any language between 1980 and November 2012 using the MeSH search terms "zinc" and "diarrhea" limited to "humans" in the following databases: Biosis, Cumulative Index to Nursing and Allied Health (CINAHL), Cochrane Central Register of Controlled Trials (CENTRAL), Embase, the WHO International Clinical Trials Registry Platform (ICTRP), Global Health, Latin American and Caribbean Health Sciences Literature (LILACS), PubMed, Scopus, Web of Science, IndMed, Egyptian Universities Library Consortium, Index Medicus for the Eastern Mediterranean Region (IMEMR), China National Knowledge Infrastructure (CNKI), WanFang, and Chinese BioMedical (CBM) database. 
Titles and abstracts were reviewed by two independent reviewers, and complete manuscripts were obtained for further review of pertinent studies. Discrepancies were resolved in consultation with a third reviewer. We restricted inclusion to individually randomized controlled trials (RCTs) of children under five years of age with acute diarrhea, including dysentery, where diarrhea was defined as the passage of at least three loose or watery stools in a $24-\mathrm{h}$ period. We excluded cluster RCTs, studies that exclusively enrolled a particular subgroup of children (e.g., HIV-infected children; preterm infants), and studies of persistent diarrhea. We included RCTs assessing oral zinc supplementation of any zinc salt in comparison to a control group receiving placebo supplement. For studies conducted in China, where placebo supplements may not have been readily available, we included trials in which cases received the same supportive therapy regardless of zinc allocation. For all studies, administration of minerals (excluding iron), vitamins, and supporting therapy beyond zinc were only considered acceptable if these were received by both the intervention and control groups. Studies that used supplements that included iron, zinc-fortified ORS, or zinc-fortified foods were excluded.

Included studies were reviewed for the following outcomes: diarrhea duration; the proportion of diarrhea episodes lasting $>3$ and $>7$ days; duration of hospitalization; duration of fever; duration of vomiting; proportion of cases vomiting; stool frequency (number per day); stool output $(\mathrm{mL})$; and death from diarrhea or any cause. Two independent reviewers entered data into structured tables, and discrepancies were resolved in consultation with a third reviewer.

We conducted independent analyses for studies assessing diarrhea due to unspecified causes and those assessing specific pathogens (e.g., rotavirus) that were laboratory confirmed prior to enrollment. All data analyses were conducted in STATA 12.0 [9]. We fit Poisson and logistic regression models to continuous and binary outcomes, respectively, weighting all outcomes by sample size. These models generated pooled estimates and 95\% confidence intervals lower bound by zero for all outcomes and upper bound by one for proportions.

For continuous outcomes, we calculated the overall percentage difference between the pooled estimates for the zinc and control groups. For binary outcomes, we calculated estimates of relative risk (RR) with placebo as the reference group and conducted random effects meta-analyses to combine RRs across studies [9].

We conducted hypothesis testing to assess the equivalence of pooled outcomes and of effect estimates by placebo and non-placebo controlled trials. To compare effect estimates, we tested the difference of mean percentage differences for continuous outcomes and the ratio of relative risks (RRR) for binary outcomes [10]. We subsequently pooled placebo and non-placebo controlled trials for outcomes with no statistically significant difference in effect size.

We assessed the association between the dose of oral zinc supplement and diarrhea duration by regressing the mean percentage difference in diarrhea duration comparing the zinc and control groups onto a categorical variable which indicated whether zinc dose was lower than, equal to, or greater than the WHO recommendation.

During the course of our analyses, we identified a zinc product called Licorzinc that appeared to be unique to China. To determine whether outcomes for Chinese studies were generalizable comparing Licorzinc to other better established zinc products, we conducted hypothesis testing to assess the equivalence of the mean percentage difference in episode duration between zinc and placebo. We also 
calculated the RRR to compare the RR of episodes lasting $>3$ days between studies using Licorzinc and other zinc products.

We plotted funnel plots to assess our primary outcomes for publication bias. We also employed the Child Health Epidemiology Reference Group (CHERG) grading system to assess the quality of evidence for each outcome on a four-point scale ("high", "moderate", "low”, "very low”) [11].

\section{Results}

The systematic literature search of the non-Chinese databases uncovered 4038 titles, and 15 were included after subsequent review of abstracts and full manuscripts for inclusion and exclusion criteria (Figure 1) [5-7,12-23]. Of the included studies, 13 were conducted in a hospital setting and two assessed episodes occurring in the community. Included studies were conducted in sites located within 10 countries: India $(n=6)$; Bangladesh $(n=5)$; Nepal $(n=1)$; Turkey $(n=1)$; Brazil $(n=1)$; Pakistan $(n=1)$; Ethiopia $(n=1)$; Yemen $(n=1)$; and Poland $(n=1)$. These studies enrolled a total of 3271 zinc-allocated and 3314 placebo-allocated diarrhea cases. The systematic literature search for Chinese studies resulted in 1520 titles, of which 89 were included (Figure 1) [24-112]. All included studies were conducted in a hospital setting, and 33 studies focused on diarrhea attributable to laboratory confirmed rotavirus. None of the included studies identified through the Chinese database were placebo-controlled; for Chinese studies, zinc and control groups received a range of supportive treatments, including fluid infusion, probiotics and antivirals. The total enrolment of included Chinese studies was 6198 zinc group and 6039 control group diarrhea cases. Table 1 describes the trial setting, sample size, and zinc intervention for all included studies.

Figure 1. Results of systematic literature search and review.

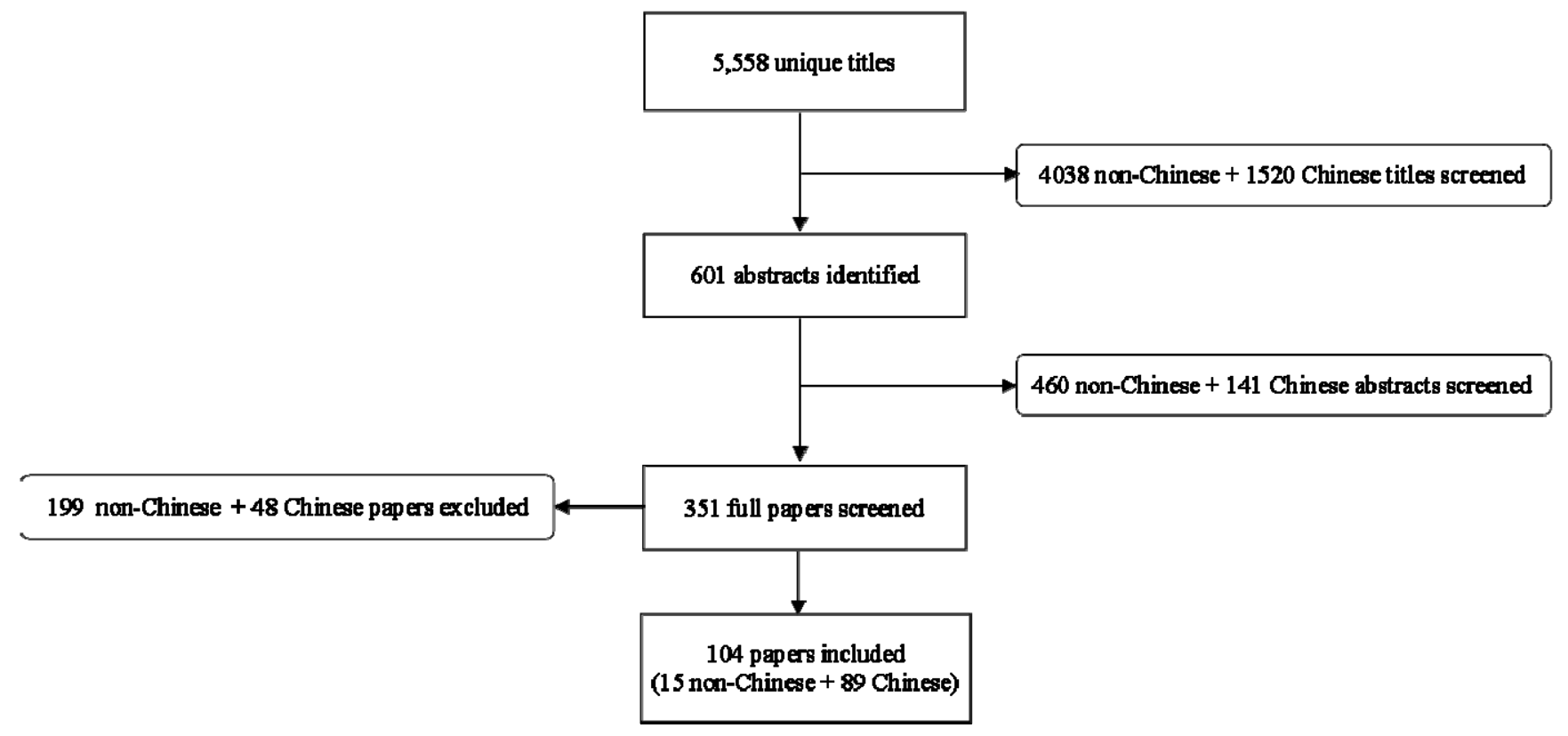


Table 1. Characteristics of included studies.

\begin{tabular}{|c|c|c|c|c|c|c|c|c|c|c|c|}
\hline $\begin{array}{c}\text { Author } \\
\text { [Reference] }\end{array}$ & $\begin{array}{c}\text { Year } \\
\text { Published }\end{array}$ & Country & $\begin{array}{c}\text { Trial } \\
\text { Setting }\end{array}$ & $\begin{array}{c}\text { Specific } \\
\text { Causative } \\
\text { Organisms } \\
\end{array}$ & $\begin{array}{l}\text { Age Group } \\
\text { (months) }\end{array}$ & $\begin{array}{l}\text { Sam } \\
\text { Zinc } \\
\text { Group }\end{array}$ & $\begin{array}{c}\text { le Size } \\
\text { Control } \\
\text { Group }\end{array}$ & Zinc Salt & $\begin{array}{c}\text { Tablet } \\
\text { or } \\
\text { Syrup }\end{array}$ & Daily Zinc Dose & $\begin{array}{c}\text { Length of } \\
\text { Supplementation } \\
\text { (days) }\end{array}$ \\
\hline Al Sonboli [17] & 2003 & Brazil & Hospital & Unknown & $3-60$ & 37 & 37 & Not Listed & Tablet & $\begin{array}{l}3-5 \text { mos: } 22.5 \mathrm{mg} \\
6-60 \mathrm{mos}: 45 \mathrm{mg}\end{array}$ & 5 \\
\hline Bahl [7] & 2002 & India & Community & Unknown & $6-35$ & 404 & 401 & Zinc Gluconate & Syrup & $\begin{array}{l}6-11 \mathrm{mos}: 15 \mathrm{mg} \\
12-35 \mathrm{mos}: 30 \mathrm{mg}\end{array}$ & 14 \\
\hline Brooks [16] & 2005 & Bangladesh & Hospital & Unknown & $1-6$ & 91 & 93 & Zinc Acetate & Syrup & $20 \mathrm{mg}$ & Duration of episode \\
\hline Brooks [16] & 2005 & Bangladesh & Hospital & Unknown & $1-6$ & 91 & 93 & Zinc Acetate & Syrup & $5 \mathrm{mg}$ & Duration of episode \\
\hline Dutta [23] & 2011 & India & Hospital & Unknown & $6-24$ & 44 & 41 & Not Listed & Syrup & $40 \mathrm{mg}$ & 14 \\
\hline Elnemr [21] & 2007 & Yemen & Hospital & Unknown & $3-24$ & 88 & 92 & Zinc Acetate & Syrup & $20 \mathrm{mg}$ & 14 \\
\hline Faruque [12] & 1999 & Bangladesh & Hospital & Unknown & $6-24$ & 343 & 341 & Zinc Acetate & Syrup & $14.2 \mathrm{mg}$ & 15 \\
\hline Fischer Walker [19] & 2006 & Pakistan & Hospital & Unknown & $1-5$ & 281 & 279 & Zinc Sulfate & Tablet & $10 \mathrm{mg}$ & 14 \\
\hline Fischer Walker [19] & 2006 & India & Hospital & Unknown & $1-5$ & 186 & 187 & Zinc Sulfate & Tablet & $10 \mathrm{mg}$ & 14 \\
\hline Fischer Walker [19] & 2006 & Ethiopia & Hospital & Unknown & $1-5$ & 87 & 90 & Zinc Sulfate & Tablet & $10 \mathrm{mg}$ & 14 \\
\hline Larson [18] & 2005 & Bangladesh & Hospital & Unknown & $3-59$ & 267 & 266 & Zinc Sulfate & Tablet & $20 \mathrm{mg}$ & 10 \\
\hline Patel [20] & 2009 & India & Hospital & Unknown & $6-59$ & 264 & 271 & Zinc Sulfate & Syrup & $20 \mathrm{mg}$ & 14 \\
\hline Patro [22] & 2010 & Poland & Hospital & Unknown & $3-48$ & 81 & 79 & Zinc Sulfate & Syrup & $\begin{array}{l}\text { 3-5 mos: } 10 \mathrm{mg} \\
\text { 6-48 mos: } 20 \mathrm{mg}\end{array}$ & 10 \\
\hline Polat [15] & 2003 & Turkey & Hospital & Unknown & $2-29$ & 52 & 54 & Zinc Sulfate & Syrup & $20 \mathrm{mg}$ & 10 \\
\hline Roy [13] & 1999 & Bangladesh & Hospital & Unknown & $3-24$ & 32 & 35 & Zinc Acetate & Syrup & $20 \mathrm{mg}$ & 14 \\
\hline Sachdev [5] & 1988 & India & Hospital & Unknown & $6-18$ & 25 & 25 & Zinc Sulfate & Tablet & $40 \mathrm{mg}$ & Not Listed \\
\hline Sazawal [6] & 1995 & India & Hospital & Unknown & $6-35$ & 456 & 481 & Zinc Gluconate & Syrup & $20 \mathrm{mg}$ & Not Listed \\
\hline Strand [14] & 2002 & Nepal & Community & Unknown & $6-35$ & 442 & 449 & Not Listed & Syrup & $\begin{array}{l}6-11 \mathrm{mos}: 15 \mathrm{mg} \\
12-35 \mathrm{mos}: 30 \mathrm{mg}\end{array}$ & $\begin{array}{l}\text { From enrolment } \\
\text { until } 7 \text { days after } \\
\text { episode subsided }\end{array}$ \\
\hline
\end{tabular}


Table 1. Cont.

\begin{tabular}{|c|c|c|c|c|c|c|c|c|c|c|c|}
\hline Zhao [24] & 2011 & China & Hospital & Unknown & $4-36$ & 40 & 40 & Licorzinc & Tablet & $\begin{array}{r}\text { 4-5 mos: } 10.8 \mathrm{mg} \\
\text { 6-12 mos: } 14.4 \mathrm{mg} \\
13-36 \text { mos: } 21.6 \mathrm{mg}\end{array}$ & Not Listed \\
\hline Zhang [25] & 2009 & China & Hospital & Rotavirus & $6-24$ & 60 & 60 & Zinc Gluconate & $\begin{array}{c}\text { Not } \\
\text { Listed }\end{array}$ & $20 \mathrm{mg}$ & Duration of episode \\
\hline Lin [26] & 2010 & China & Hospital & Rotavirus & $1.5-36$ & 58 & 58 & Zinc Gluconate & Syrup & $\begin{array}{l}\text { 1.5-5 mos: } 10 \mathrm{mg} \\
\text { 6-36 mos: } 20 \mathrm{mg}\end{array}$ & Duration of episode \\
\hline Zhou [27] & 2010 & China & Hospital & Rotavirus & $6-24$ & 42 & 40 & Zinc Gluconate & $\begin{array}{c}\text { Not } \\
\text { Listed }\end{array}$ & $20 \mathrm{mg}$ & 14 \\
\hline Yang [28] & 2011 & China & Hospital & Unknown & $3-36$ & 42 & 40 & Zinc Gluconate & Tablet & $\begin{array}{l}3-5 \text { mos: } 10 \mathrm{mg} \\
6-36 \text { mos: } 20 \mathrm{mg}\end{array}$ & $10-14$ \\
\hline Liu [29] & 2010 & China & Hospital & Unknown & $5-18$ & 40 & 40 & Zinc Gluconate & $\begin{array}{c}\text { Not } \\
\text { Listed }\end{array}$ & $\begin{array}{c}5 \text { mos: } 10 \mathrm{mg} \\
6-18 \mathrm{mos}: 20 \mathrm{mg}\end{array}$ & $10-14$ \\
\hline Chen [30] & 2006 & China & Hospital & Rotavirus & $0-24$ & 30 & 30 & Zinc gluconate & $\begin{array}{c}\text { Not } \\
\text { Listed }\end{array}$ & $10 \mathrm{mg}$ & Not Listed \\
\hline Liu [31] & 2011 & China & Hospital & Unknown & $6.8-22$ & 90 & 90 & Zinc Gluconate & Tablet & $20 \mathrm{mg}$ & Not Listed \\
\hline Liu [32] & 2009 & China & Hospital & Unknown & $6-36$ & 112 & 108 & Zinc Gluconate & Tablet & $20 \mathrm{mg}$ & 10 \\
\hline $\mathrm{Fu}[33]$ & 2010 & China & Hospital & Rotavirus & $2-24$ & 98 & 102 & Zinc Gluconate & Syrup & $5 \mathrm{mg}$ & Not Listed \\
\hline Zhou [34] & 2008 & China & Hospital & Unknown & $2-48$ & 40 & 40 & Licorzinc & $\begin{array}{c}\text { Not } \\
\text { Listed }\end{array}$ & $\begin{array}{c}2-5 \text { mos: } 7.5 \mathrm{mg} \\
\text { 6-12 mos: } 11.25 \mathrm{mg} \\
\text { 13-48 mos: } 18.75 \mathrm{mg}\end{array}$ & $10-14$ \\
\hline Chen [35] & 2008 & China & Hospital & Rotavirus & $4-48$ & 60 & 60 & Licorzinc & $\begin{array}{c}\text { Not } \\
\text { Listed }\end{array}$ & $\begin{array}{c}4-5 \text { mos: } 7.2 \mathrm{mg} \\
\text { 6-48 mos: } 10.8 \mathrm{mg}\end{array}$ & Not Listed \\
\hline
\end{tabular}


Table 1. Cont.

\begin{tabular}{|c|c|c|c|c|c|c|c|c|c|c|c|}
\hline Guan [36] & 2012 & China & Hospital & Rotavirus & $1.5-45.6$ & 45 & 45 & Licorzinc & $\begin{array}{c}\text { Not } \\
\text { Listed }\end{array}$ & $\begin{array}{c}1.5-5 \text { mos: } 7.5 \mathrm{mg} \\
\text { 6-11 mos: } 11.25 \mathrm{mg} \\
12-45.6 \mathrm{mos}: \\
18.75 \mathrm{mg}\end{array}$ & $10-14$ \\
\hline $\mathrm{Wu}$ [37] & 2010 & China & Hospital & Rotavirus & $4-13$ & 46 & 46 & Licorzinc & $\begin{array}{c}\text { Not } \\
\text { Listed }\end{array}$ & $\begin{array}{l}\text { 4-5 mos: } 10 \mathrm{mg} \\
6-13 \mathrm{mos}: 20 \mathrm{mg}\end{array}$ & Not Listed \\
\hline Zhou [38] & 2010 & China & Hospital & Unknown & $6-24$ & 65 & 60 & Licorzinc & Tablet & $20 \mathrm{mg}$ & Not Listed \\
\hline Luo [39] & 2009 & China & Hospital & rotavirus & $6-36$ & 55 & 50 & Licorzinc & Tablet & $18.75 \mathrm{mg}$ & Not Listed \\
\hline Zhang [40] & 2010 & China & Hospital & Unknown & $5-48$ & 50 & 50 & Licorzinc & $\begin{array}{c}\text { Not } \\
\text { Listed }\end{array}$ & Not Listed * & Not Listed \\
\hline $\mathrm{Ju}[41]$ & 2007 & China & Hospital & Unknown & $6-36$ & 40 & 38 & Licorzinc & Tablet & $\begin{array}{c}6-12 \text { mos: } 11-25 \mathrm{mg} \\
13-36 \text { mos: } 15 \mathrm{mg}\end{array}$ & Not Listed \\
\hline Wang [42] & 2012 & China & Hospital & Unknown & $6-36$ & 30 & 30 & Licorzinc & Tablet & Not Listed * & 3 \\
\hline Hong [43] & 2009 & China & Hospital & Rotavirus & $3-60$ & 140 & 120 & Zinc Sulfate & Syrup & $\begin{array}{l}\text { 3-11 mos: } 20 \mathrm{mg} \\
\text { 12-36 mos: } 30 \mathrm{mg} \\
37-60 \text { mos: } 40 \mathrm{mg}\end{array}$ & Not Listed \\
\hline Lin [44] & 1994 & China & Hospital & Unknown & $0.5-24$ & 46 & 58 & Zinc Sulfate & Syrup & $10-14 \mathrm{mg} / \mathrm{kg}$ * & Not Listed \\
\hline Yan [45] & 2011 & China & Hospital & Unknown & $5-36$ & 70 & 50 & Zinc Sulfate & Syrup & $\begin{array}{c}5 \text { mos: } 50 \mathrm{mg} \\
\text { 6-36 mos: } 100 \mathrm{mg}\end{array}$ & Not Listed \\
\hline $\mathrm{He}[46]$ & 1997 & China & Hospital & Unknown & $6-36$ & 52 & 58 & Zinc Gluconate & $\begin{array}{c}\text { Not } \\
\text { Listed }\end{array}$ & $20 \mathrm{mg}$ & Not Listed \\
\hline Wei [47] & 2011 & China & Hospital & Unknown & $3-36$ & 44 & 42 & Zinc Gluconate & Syrup & $\begin{array}{l}3-5 \text { mos: } 10 \mathrm{mg} \\
6-36 \text { mos: } 20 \mathrm{mg}\end{array}$ & $10-14$ \\
\hline Yang [48] & 2012 & China & Hospital & Unknown & $0-36$ & 80 & 80 & Zinc Gluconate & Tablet & $\begin{array}{c}0-5 \text { mos: } 10 \mathrm{mg} \\
\text { 6-36 mos: } 20 \mathrm{mg}\end{array}$ & 10 \\
\hline
\end{tabular}


Table 1. Cont.

\begin{tabular}{|c|c|c|c|c|c|c|c|c|c|c|c|}
\hline $\mathrm{Pu}[49]$ & 2010 & China & Hospital & Rotavirus & $0-24$ & 38 & 34 & Zinc Gluconate & $\begin{array}{c}\text { Not } \\
\text { Listed }\end{array}$ & $\begin{array}{l}0-5 \text { mos: } 10 \mathrm{mg} \\
6-24 \text { mos: } 20 \mathrm{mg}\end{array}$ & Not Listed \\
\hline Zhang [50] & 2011 & China & Hospital & Rotavirus & $3-36$ & 53 & 53 & Zinc Gluconate & $\begin{array}{c}\text { Not } \\
\text { Listed }\end{array}$ & $\begin{array}{l}3-5 \text { mos: } 10 \mathrm{mg} \\
6-36 \text { mos: } 20 \mathrm{mg}\end{array}$ & 10 \\
\hline Sun [51] & 2008 & China & Hospital & Unknown & $1.5-36$ & 45 & 45 & Zinc Gluconate & Syrup & $\begin{array}{l}1.5-5 \mathrm{mos}: 10 \mathrm{mg} \\
6-36 \mathrm{mos}: 20 \mathrm{mg}\end{array}$ & Not Listed \\
\hline Zhang [52] & 2011 & China & Hospital & Unknown & $3-36$ & 90 & 90 & Zinc Gluconate & Syrup & $\begin{array}{l}3-5 \text { mos: } 10 \mathrm{mg} \\
6-36 \mathrm{mos}: 20 \mathrm{mg}\end{array}$ & Not Listed \\
\hline $\operatorname{Lin}[53]$ & 2010 & China & Hospital & Rotavirus & $6-54$ & 28 & 20 & Zinc Gluconate & Tablet & 6-54 mos: $20 \mathrm{mg}$ & 14 \\
\hline Liu [54] & 2009 & China & Hospital & Unknown & $3-36$ & 95 & 91 & Zinc Gluconate & $\begin{array}{c}\text { Not } \\
\text { Listed }\end{array}$ & $\begin{array}{l}3-5 \text { mos: } 10 \mathrm{mg} \\
6-36 \mathrm{mos}: 20 \mathrm{mg}\end{array}$ & $10-14$ \\
\hline Qiao [55] & 2011 & China & Hospital & Unknown & $6-36$ & 73 & 72 & Zinc Gluconate & Tablet & 6-36 mos: $20 \mathrm{mg}$ & 14 \\
\hline Zhang [56] & 2007 & China & Hospital & Unknown & $0-24$ & 85 & 90 & Zinc Gluconate & $\begin{array}{c}\text { Not } \\
\text { Listed }\end{array}$ & $\begin{array}{l}0-5 \text { mos: } 10 \mathrm{mg} \\
6-24 \mathrm{mos}: 20 \mathrm{mg}\end{array}$ & 10 \\
\hline Zhao [57] & 2012 & China & Hospital & Unknown & $0-24$ & 70 & 70 & Zinc Gluconate & Syrup & $\begin{array}{l}0-5 \text { mos: } 10 \mathrm{mg} \\
6-24 \mathrm{mos}: 20 \mathrm{mg}\end{array}$ & $10-14$ \\
\hline Cai [58] & 2011 & China & Hospital & Unknown & $0-24$ & 88 & 84 & Zinc Gluconate & $\begin{array}{c}\text { Not } \\
\text { Listed }\end{array}$ & $\begin{array}{l}0-5 \text { mos: } 10 \mathrm{mg} \\
6-24 \mathrm{mos}: 20 \mathrm{mg}\end{array}$ & 14 \\
\hline Zhang [59] & 2012 & China & Hospital & Rotavirus & $6-17$ & 120 & 120 & Zinc Gluconate & Tablet & $20 \mathrm{mg}$ & $10-14$ \\
\hline Qiao [60] & 2012 & China & Hospital & Unknown & $0-24$ & 85 & 85 & Zinc Gluconate & $\begin{array}{c}\text { Not } \\
\text { Listed }\end{array}$ & $\begin{array}{l}0-5 \text { mos: } 10 \mathrm{mg} \\
6-24 \mathrm{mos}: 20 \mathrm{mg}\end{array}$ & 10 \\
\hline Zhong [61] & 2012 & China & Hospital & Rotavirus & $3-48$ & 50 & 50 & Zinc Gluconate & Tablet & $\begin{array}{l}3-5 \text { mos: } 10 \mathrm{mg} \\
6-48 \mathrm{mos}: 20 \mathrm{mg}\end{array}$ & 10 \\
\hline Wang [62] & 2011 & China & Hospital & Rotavirus & $0-24$ & 60 & 60 & Zinc Gluconate & $\begin{array}{c}\text { Not } \\
\text { Listed }\end{array}$ & $\begin{array}{l}0-5 \text { mos: } 10 \mathrm{mg} \\
6-24 \mathrm{mos}: 20 \mathrm{mg}\end{array}$ & 10 \\
\hline Yang [63] & 2008 & China & Hospital & Rotavirus & $0-36$ & 164 & 168 & Zinc Gluconate & $\begin{array}{c}\text { Not } \\
\text { Listed }\end{array}$ & $\begin{array}{l}0-5 \mathrm{mos}: 10 \mathrm{mg} \\
6-36 \mathrm{mos}: 20 \mathrm{mg}\end{array}$ & 10 \\
\hline
\end{tabular}


Table 1. Cont.

\begin{tabular}{|c|c|c|c|c|c|c|c|c|c|c|c|}
\hline Zhao [64] & 2012 & China & Hospital & Rotavirus & $6-36$ & 60 & 60 & Zinc Gluconate & Syrup & $35 \mathrm{mg}$ & 10 \\
\hline $\mathrm{Ma}[65]$ & 2012 & China & Hospital & Rotavirus & $4-42$ & 41 & 41 & Zinc Gluconate & $\begin{array}{c}\text { Not } \\
\text { Listed }\end{array}$ & $20 \mathrm{mg}$ & Not Listed \\
\hline Chen [66] & 2012 & China & Hospital & Rotavirus & $0-36$ & 93 & 93 & Zinc Gluconate & $\begin{array}{c}\text { Not } \\
\text { Listed }\end{array}$ & $\begin{array}{l}0-5 \text { mos: } 10 \mathrm{mg} \\
6-36 \text { mos: } 20 \mathrm{mg}\end{array}$ & 10 \\
\hline $\mathrm{Hu}[67]$ & 2009 & China & Hospital & Rotavirus & $4-36$ & 60 & 60 & Zinc Gluconate & Tablet & $\begin{array}{l}4-5 \text { mos: } 10 \mathrm{mg} \\
6-36 \mathrm{mos}: 20 \mathrm{mg}\end{array}$ & 10 \\
\hline Yuan [68] & 2011 & China & Hospital & Unknown & $1-36$ & 100 & 100 & Zinc Gluconate & Tablet & $\begin{array}{l}1-12 \text { mos: } 70 \mathrm{mg} \\
\text { 13-36 mos: } 140 \mathrm{mg}\end{array}$ & 14 \\
\hline $\operatorname{Tan}[69]$ & 2011 & China & Hospital & Unknown & $3-36$ & 50 & 35 & Zinc Gluconate & Tablet & $\begin{array}{l}3-5 \text { mos: } 10 \mathrm{mg} \\
6-36 \text { mos: } 20 \mathrm{mg}\end{array}$ & $10-14$ \\
\hline Liu [70] & 2010 & China & Hospital & Unknown & $0-36$ & 89 & 77 & Zinc Gluconate & Syrup & $\begin{array}{l}0-5 \text { mos: } 10 \mathrm{mg} \\
6-36 \text { mos: } 20 \mathrm{mg}\end{array}$ & 10 \\
\hline $\mathrm{Hu}[71]$ & 2011 & China & Hospital & Unknown & $3-60$ & 108 & 100 & Zinc Gluconate & Tablet & $\begin{array}{l}3-5 \text { mos: } 10 \mathrm{mg} \\
6-60 \mathrm{mos}: 20 \mathrm{mg}\end{array}$ & 14 \\
\hline $\mathrm{Li}[72]$ & 2008 & China & Hospital & Unknown & $6-36$ & 40 & 38 & Zinc Gluconate & Tablet & $\begin{array}{l}6-12 \text { mos: } 7.5 \mathrm{mg} \\
\text { 13-36 mos: } 15 \mathrm{mg}\end{array}$ & 3 \\
\hline Gao [73] & 2012 & China & Hospital & Unknown & $3-36$ & 74 & 74 & Zinc Gluconate & $\begin{array}{c}\text { Not } \\
\text { Listed }\end{array}$ & $\begin{array}{l}3-5 \text { mos: } 10 \mathrm{mg} \\
6-36 \text { mos: } 20 \mathrm{mg}\end{array}$ & 14 \\
\hline $\mathrm{Wu}[74]$ & 2011 & China & Hospital & Unknown & $3-60$ & 20 & 20 & Zinc Sulfate & Syrup & $10 \mathrm{mg}$ & 10 \\
\hline $\mathrm{Wu}[74]$ & 2011 & China & Hospital & Unknown & $3-60$ & 20 & 20 & Zinc Sulfate & $\begin{array}{c}\text { Not } \\
\text { Listed }\end{array}$ & $10 \mathrm{mg}$ & 10 \\
\hline Liu [75] & 2011 & China & Hospital & Unknown & $3-60$ & 54 & 53 & Zinc Gluconate & Tablet & $\begin{array}{l}3-5 \text { mos: } 10 \mathrm{mg} \\
6-60 \text { mos: } 20 \mathrm{mg}\end{array}$ & $3-5$ \\
\hline
\end{tabular}


Table 1. Cont.

\begin{tabular}{|c|c|c|c|c|c|c|c|c|c|c|c|}
\hline Chen [76] & 2010 & China & Hospital & Unknown & $5-36$ & 42 & 20 & Zinc Gluconate & $\begin{array}{c}\text { Not } \\
\text { Listed }\end{array}$ & $\begin{array}{c}5 \text { mos: } 10 \mathrm{mg} \\
\text { 6-36 mos: } 20 \mathrm{mg}\end{array}$ & $10-14$ \\
\hline Ma [77] & 2012 & China & Hospital & Unknown & $2-36$ & 63 & 63 & Zinc Gluconate & $\begin{array}{c}\text { Not } \\
\text { Listed }\end{array}$ & $\begin{array}{l}2-5 \text { mos: } 70 \mathrm{mg} \\
\text { 6-36 mos: } 140 \mathrm{mg}\end{array}$ & $10-14$ \\
\hline $\mathrm{Lu}[78]$ & 2012 & China & Hospital & Unknown & $6-18$ & 120 & 140 & Zinc Gluconate & $\begin{array}{c}\text { Not } \\
\text { Listed }\end{array}$ & $140 \mathrm{mg}$ & $10-14$ \\
\hline $\mathrm{Ma}$ [79] & 2012 & China & Hospital & Unknown & $6-36$ & 58 & 52 & Zinc Gluconate & Syrup & 6-36 mos: $20 \mathrm{mg}$ & 10 \\
\hline Ao [80] & 2012 & China & Hospital & Rotavirus & $0-24$ & 87 & 80 & Zinc Gluconate & Syrup & $\begin{array}{l}\text { 0-5 mos: } 10 \mathrm{mg} \\
\text { 6-24 mos: } 20 \mathrm{mg}\end{array}$ & Not Listed \\
\hline $\mathrm{Gu}[81]$ & 2011 & China & Hospital & Unknown & $3-60$ & 56 & 60 & Zinc Gluconate & Syrup & $\begin{array}{l}3-5 \text { mos: } 10 \mathrm{mg} \\
6-60 \text { mos: } 20 \mathrm{mg}\end{array}$ & 10 \\
\hline Wen [82] & 2006 & China & Hospital & Unknown & $0-24$ & 30 & 29 & Zinc Gluconate & $\begin{array}{c}\text { Not } \\
\text { Listed }\end{array}$ & $20 \mathrm{mg}$ & $10-14$ \\
\hline Wang [83] & 2011 & China & Hospital & Unknown & $3-36$ & 60 & 60 & Licorzinc & $\begin{array}{c}\text { Not } \\
\text { Listed }\end{array}$ & $10-20 \mathrm{mg}$ * & Duration of episode \\
\hline Liu [84] & 2012 & China & Hospital & Rotavirus & $8-30$ & 90 & 90 & Licorzinc & $\begin{array}{c}\text { Not } \\
\text { Listed }\end{array}$ & 8-30 mos: $20 \mathrm{mg}$ & Not Listed \\
\hline Liu [85] & 2012 & China & Hospital & Unknown & $3-60$ & 100 & 100 & Licorzinc & Tablet & $\begin{array}{l}\text { 3-5 mos: } 10 \mathrm{mg} \\
\text { 6-60 mos: } 20 \mathrm{mg}\end{array}$ & Not Listed \\
\hline Tong [86] & 2011 & China & Hospital & Unknown & $2-36$ & 98 & 98 & Licorzinc & $\begin{array}{c}\text { Not } \\
\text { Listed }\end{array}$ & $\begin{array}{l}\text { 2-5 mos: } 10 \mathrm{mg} \\
\text { 6-36 mos: } 20 \mathrm{mg}\end{array}$ & Not Listed \\
\hline Qiu [87] & 2010 & China & Hospital & Rotavirus & $1-24$ & 53 & 52 & Licorzinc & Tablet & $\begin{array}{l}\text { 1-5 mos: } 10 \mathrm{mg} \\
\text { 6-24 mos: } 20 \mathrm{mg}\end{array}$ & 14 \\
\hline Kong [88] & 2011 & China & Hospital & Unknown & $3-30$ & 35 & 35 & Zinc Gluconate & Tablet & $\begin{array}{c}3-5 \text { mos: } 10 \mathrm{mg} \\
\text { 6-11 mos: } 15 \mathrm{mg} \\
\text { 12-30 mos: } 20 \mathrm{mg}\end{array}$ & 14 \\
\hline $\mathrm{He}$ [89] & 2007 & China & Hospital & Rotavirus & $5-22$ & 60 & 63 & Zinc Gluconate & $\begin{array}{c}\text { Not } \\
\text { Listed }\end{array}$ & $20 \mathrm{mg}$ & Not Listed \\
\hline
\end{tabular}


Table 1. Cont.

\begin{tabular}{|c|c|c|c|c|c|c|c|c|c|c|c|}
\hline Kang [90] & 2010 & China & Hospital & Rotavirus & $6-36$ & 92 & 80 & Zinc Gluconate & Tablet & $20 \mathrm{mg}$ & 14 \\
\hline $\mathrm{Su}[91]$ & 2012 & China & Hospital & Rotavirus & $6-36$ & 97 & 97 & Zinc Gluconate & $\begin{array}{c}\text { Not } \\
\text { Listed }\end{array}$ & $20 \mathrm{mg}$ & Not Listed \\
\hline Huang [92] & 2010 & China & Hospital & Rotavirus & $2-36$ & 100 & 100 & Not Listed & Tablet & $\begin{array}{l}2-5 \text { mos: } 10 \mathrm{mg} \\
\text { 6-36 mos: } 20 \mathrm{mg}\end{array}$ & Not Listed \\
\hline Zhang [93] & 2006 & China & Hospital & Unknown & $0-36$ & 83 & 63 & Licorzinc & Syrup & $\begin{array}{l}\text { 0-5 mos: } 10 \mathrm{mg} \\
\text { 6-36 mos: } 20 \mathrm{mg}\end{array}$ & $10-14$ \\
\hline Wang [94] & 2012 & China & Hospital & Unknown & $4-30$ & 60 & 60 & Zinc Gluconate & Syrup & $10 \mathrm{mg}$ & Not Listed \\
\hline Lin [95] & 2008 & China & Hospital & Unknown & $0.5-34$ & 60 & 60 & Zinc Gluconate & Tablet & $\begin{array}{l}0.5-5 \text { mos: } 140 \mathrm{mg} \\
6-34 \text { mos: } 280 \mathrm{mg}\end{array}$ & $10-14$ \\
\hline Yan [96] & 2011 & China & Hospital & Unknown & $6-60$ & 57 & 57 & Zinc Gluconate & Tablet & $20 \mathrm{mg}$ & 10 \\
\hline $\mathrm{Yu}[97]$ & 2012 & China & Hospital & Unknown & $0-36$ & 40 & 40 & Zinc Gluconate & Tablet & $\begin{array}{l}0-5 \text { mos: } 10 \mathrm{mg} \\
\text { 6-36 mos: } 20 \mathrm{mg}\end{array}$ & $10-14$ \\
\hline Zhang [98] & 2011 & China & Hospital & Rotavirus & $4-36$ & 128 & 128 & Zinc Gluconate & Syrup & $\begin{array}{l}\text { 4-5 mos: } 10 \mathrm{mg} \\
\text { 6-36 mos: } 20 \mathrm{mg}\end{array}$ & 14 \\
\hline $\mathrm{Xu}[99]$ & 2010 & China & Hospital & Rotavirus & $2-36$ & 84 & 83 & Zinc Gluconate & $\begin{array}{c}\text { Not } \\
\text { Listed }\end{array}$ & $\begin{array}{l}\text { 2-5 mos: } 10 \mathrm{mg} \\
\text { 6-36 mos: } 20 \mathrm{mg}\end{array}$ & 14 \\
\hline Tan [100] & 2010 & China & Hospital & Unknown & $3.5-60$ & 55 & 55 & Zinc Gluconate & Syrup & $\begin{array}{l}3.5-5 \text { mos: } 10 \mathrm{mg} \\
6-60 \mathrm{mos}: 20 \mathrm{mg}\end{array}$ & $10-14$ \\
\hline Shen [101] & 2012 & China & Hospital & Rotavirus & $2.5-40$ & 46 & 42 & Zinc Gluconate & $\begin{array}{c}\text { Not } \\
\text { Listed }\end{array}$ & $\begin{array}{l}2.5-5 \text { mos: } 10 \mathrm{mg} \\
6-40 \text { mos: } 20 \mathrm{mg}\end{array}$ & Duration of episode \\
\hline Wang [102] & 2010 & China & Hospital & Unknown & $6-48$ & 52 & 51 & Zinc Gluconate & Tablet & $20 \mathrm{mg}$ & Not Listed \\
\hline
\end{tabular}


Table 1. Cont.

\begin{tabular}{|c|c|c|c|c|c|c|c|c|c|c|c|}
\hline Chen [103] & 2011 & China & Hospital & Unknown & $1-36$ & 50 & 50 & Zinc Gluconate & Tablet & $\begin{array}{l}\text { 1-5 mos: } 5 \mathrm{mg} \\
\text { 6-36 mos: } 10 \mathrm{mg}\end{array}$ & Not Listed \\
\hline Meng [104] & 2012 & China & Hospital & Unknown & $0-24$ & 90 & 90 & Zinc Gluconate & Tablet & $\begin{array}{c}0-5 \text { mos: } 2.5 \mathrm{mg} \\
\text { 6-12 mos: } 5 \mathrm{mg} \\
\text { 13-24 mos: } 10 \mathrm{mg}\end{array}$ & Not Listed \\
\hline Zhong [105] & 2010 & China & Hospital & Unknown & $1-24$ & 60 & 60 & Zinc Gluconate & Tablet & $\begin{array}{c}1-5 \text { mos: } 2.5 \mathrm{mg} \\
\text { 6-12 mos: } 5 \mathrm{mg} \\
\text { 13-24 mos: } 7.5 \mathrm{mg}\end{array}$ & $5-7$ \\
\hline Xie [106] & 2010 & China & Hospital & Rotavirus & $6-36$ & 128 & 124 & Zinc Gluconate & Tablet & $20 \mathrm{mg}$ & Not Listed \\
\hline Fan [107] & 2012 & China & Hospital & Unknown & $0-36$ & 163 & 121 & Not Listed & $\begin{array}{c}\text { Not } \\
\text { Listed }\end{array}$ & $\begin{array}{l}\text { 0-5 mos: } 10 \mathrm{mg} \\
\text { 6-36 mos: } 20 \mathrm{mg}\end{array}$ & 10 \\
\hline Zhou [108] & 2012 & China & Hospital & Rotavirus & $6-24$ & 75 & 75 & Zinc Gluconate & Syrup & $20 \mathrm{mg}$ & $10-14$ \\
\hline Zhao [109] & 2008 & China & Hospital & Unknown & $0-36$ & 44 & 43 & Zinc Gluconate & Tablet & $\begin{array}{l}0-5 \mathrm{mos}: 10 \mathrm{mg} \\
6-24 \mathrm{mos}: 20 \mathrm{mg}\end{array}$ & Not Listed \\
\hline Wan [110] & 2006 & China & Hospital & Unknown & $6-36$ & 26 & 24 & Not Listed & $\begin{array}{c}\text { Not } \\
\text { Listed }\end{array}$ & Not Listed & Not Listed \\
\hline Yang [111] & 2012 & China & Hospital & Unknown & $6-60$ & 60 & 60 & Not Listed & $\begin{array}{c}\text { Not } \\
\text { Listed }\end{array}$ & $20 \mathrm{mg}$ & Not Listed \\
\hline Luo [112] & 2012 & China & Hospital & Unknown & $0-36$ & 168 & 196 & Not Listed & $\begin{array}{c}\text { Not } \\
\text { Listed }\end{array}$ & $\begin{array}{l}\text { 0-5 mos: } 10 \mathrm{mg} \\
\text { 6-36 mos: } 20 \mathrm{mg}\end{array}$ & Not Listed \\
\hline
\end{tabular}

* Study not included in dose analyses. 
The results of the studies identified through non-Chinese databases are summarized in Tables 2 and 3. Acute episodes were $4 \%$ (95\% CI: $1 \%-8 \%)$ shorter in duration among children treated with zinc compared to those receiving placebo (Table 2). Among children hospitalized for diarrhea, the duration of hospitalization was reduced by $37 \%$ (95\% CI: $21 \%-53 \%$ ) comparing the zinc and control groups (Table 2). Stool frequency was decreased by 6\% (95\% CI: 2\%-10\%) among zinc-treated children. Zinc-treated children had a reduced relative risk (RR) of acute diarrhea lasting beyond three and seven days and an increased risk of vomiting (RR: 1.83; 95\% CI: 1.40-2.39) (Table 3).

Table 2. Pooled means of select outcomes for non-Chinese studies.

\begin{tabular}{ccccc}
\hline Outcome & $\begin{array}{c}\text { Study } \\
\text { Sites }^{1}\end{array}$ & \multicolumn{2}{c}{$\begin{array}{c}\text { Pooled Mean } \\
(\mathbf{9 5 \%} \text { CI) }\end{array}$} & Percent Difference $^{\mathbf{3}}$ \\
\cline { 2 - 5 } & $\boldsymbol{N}$ & Zinc Group & Control Group & $(\%)$ \\
\hline $\begin{array}{c}\text { Duration of } \\
\text { Episode (days) } \\
\text { Duration of }\end{array}$ & 13 & $3.51(3.43-3.60)$ & $3.67(3.59-3.76)$ & $-4.4(-7.8,-1.0)$ \\
$\begin{array}{c}\text { Hospitalization (days) } \\
\text { Stool Output (mL) } \\
\text { Stool Frequency }\end{array}$ & 1 & $2.00(1.99-2.01)$ & $3.17(2.38-3.96)$ & $-36.9(-52.6,-21.2)$ \\
(Number per day) & 6 & $391.2(388.5-393.8)$ & $388.8(386.2-391.5)$ & $0.6(-0.3,1.6)$ \\
\hline
\end{tabular}

${ }^{1}$ Individual studies may contribute more than one study site $(N)$ to each estimate $;{ }^{2}$ Estimates for $\geq 2$ study sites generated by Poisson regression model weighted by sample size; ${ }^{3}$ Percent difference calculated by: $100 \times(($ Pooled Zinc Estimate - Pooled Control Estimate)/Pooled Control Estimate); 95\% CI calculated by: Percent Difference $\pm 1.96 \times\left\{\mid\left(\right.\right.$ mean $_{\text {zinc }} /$ mean $\left._{\text {control }}\right) \mid \times \operatorname{sqrt}\left[(\text { std error } \text { zinc })^{2} /\left(\text { mean }_{\text {zinc }}\right)^{2}+\left(\text { std error }_{\text {control }}\right)^{2} /\right.$ $\left.\left.\left(\text { mean }_{\text {control }}\right)^{2}\right]\right\} \times 100$.

Table 3. Pooled relative risk of select outcomes for non-Chinese studies.

\begin{tabular}{ccccc}
\hline Outcome & $\begin{array}{c}\text { Study } \\
\text { Sites }\end{array}$ & \multicolumn{2}{c}{$\begin{array}{c}\text { Pooled Estimate } \\
\text { Percentage (95\% CI) }\end{array}$} & \multirow{2}{*}{ Pooled Relative Risk $^{\mathbf{3}}$} \\
& $\boldsymbol{N}$ & Zinc Group & Control Group & RR (95\% CI) \\
\hline Episodes > 3 days (\%) & 3 & $29.7(26.7-32.7)$ & $39.5(36.3-42.7)$ & $0.78(0.67-0.90)$ \\
Episodes > 7 days (\%) & 6 & $10.3(8.9-11.7)$ & $14.9(13.2-16.5)$ & $0.74(0.55-0.99)$ \\
Vomiting (\%) & 3 & $18.8(16.0-21.6)$ & $9.4(7.3-11.4)$ & $1.83(1.40-2.39)$ \\
\hline
\end{tabular}

${ }^{1}$ Individual studies may contribute more than one study site $(N)$ to each estimate; ${ }^{2}$ Estimates for $\geq 2$ study sites generated by logistic regression model weighted by sample size; ${ }^{3}$ Estimates for $\geq 2$ studies generated by random effects meta-analysis.

Outcomes pooled across studies conducted in China showed reductions in the duration of diarrhea, hospitalization, fever, vomiting, stool output and stool frequency among zinc-treated children with acute diarrhea attributable to rotavirus and to non-specific causes (Table 4). The reduction in the duration of diarrhea was 37\% (95\% CI: 35\%-39\%) among non-specific episodes and 31\% (95\% CI: 29\%-34\%) among rotavirus episodes (Table 4). The RR of diarrhea lasting beyond three days was reduced among zinc-treated patients with non-specific (RR: 0.73 ; 95\% CI: 0.66-0.79) and rotavirus (RR: 0.70; 95\% CI: 0.63-0.78) diarrhea (Table 5; Figures 2 and 3). 
Table 4. Pooled means of select outcomes for Chinese studies.

\begin{tabular}{|c|c|c|c|c|c|}
\hline \multirow[t]{2}{*}{ Outcome } & \multirow{2}{*}{$\begin{array}{c}\text { Specific } \\
\text { Causative } \\
\text { Pathogens } \\
\end{array}$} & \multirow{2}{*}{$\begin{array}{c}\text { Study } \\
\text { Sites }{ }^{1} \\
N \\
\end{array}$} & \multicolumn{2}{|c|}{$\begin{array}{l}\text { Pooled Mean } \\
(95 \% \mathrm{CI})^{2}\end{array}$} & \multirow{2}{*}{$\begin{array}{c}\text { Percent Difference } \\
\text { (\%) }\end{array}$} \\
\hline & & & Zinc Group & Control Group & \\
\hline \multirow{2}{*}{$\begin{array}{c}\text { Duration of } \\
\text { Episode (days) }\end{array}$} & Unknown & 40 & $2.96(2.90-3.03)$ & $4.68(4.60-4.77)$ & $-36.8(-38.7,-34.8)$ \\
\hline & Rotavirus & 24 & $3.45(3.36-3.54)$ & $5.01(4.89-5.12)$ & $-31.1(-33.5,-28.8)$ \\
\hline \multirow{2}{*}{$\begin{array}{c}\text { Duration of } \\
\text { Hospitalization } \\
\text { (days) }\end{array}$} & Unknown & 10 & $4.65(4.50-4.80)$ & $6.43(6.25-6.61)$ & $-27.7(-30.8,-24.6)$ \\
\hline & Rotavirus & 2 & $4.15(3.79-4.51)$ & $6.1(5.66-6.54)$ & $-32.0(-39.6,-24.3)$ \\
\hline \multirow{2}{*}{$\begin{array}{l}\text { Duration of } \\
\text { Fever (days) }\end{array}$} & Unknown & 13 & $1.90(1.80-1.99)$ & $2.81(2.70-2.92)$ & $-32.4(-36.5,-28.2)$ \\
\hline & Rotavirus & 4 & $1.96(1.78-2.14)$ & $3.18(2.95-3.41)$ & $-38.4(-45.6,-31.2)$ \\
\hline \multirow{2}{*}{$\begin{array}{c}\text { Duration of } \\
\text { Vomiting (days) }\end{array}$} & Unknown & 6 & $1.15(1.05-1.25)$ & $1.53(1.41-1.64)$ & $-24.8(-33.3,-16.4)$ \\
\hline & Rotavirus & 3 & $1.84(1.64-2.04)$ & $2.49(2.26-2.72)$ & $-26.1(-36.6,-15.6)$ \\
\hline \multirow{2}{*}{ Stool Output (mL) } & Unknown & 1 & $40(38.1-41.9)$ & $70(68.0-72.0)$ & $-42.9(-46.0,-39.7)$ \\
\hline & Rotavirus & 1 & $278.4(256.8-300.0)$ & $425.4(382.1-468.7)$ & $-34.6(-42.9,-26.2)$ \\
\hline \multirow{2}{*}{$\begin{array}{l}\text { Stool Frequency } \\
\text { (Number per day) }\end{array}$} & Unknown & 1 & $4(3.8-4.2)$ & $8(7.6-8.4)$ & $-50.0(-53.5,-46.5)$ \\
\hline & Rotavirus & 2 & $3.74(3.30-4.18)$ & $4.27(3.77-4.77)$ & $-12.4(-27.0,2.1)$ \\
\hline
\end{tabular}

${ }^{1}$ Individual studies may contribute more than one study site $(N)$ to each estimate; ${ }^{2}$ Estimates for $\geq 2$ study sites generated by Poisson regression model weighted by sample size; ${ }^{3}$ Percent difference calculated by: $100 \times(($ Pooled Zinc Estimate - Pooled Control Estimate)/Pooled Control Estimate); 95\% CI calculated by: Percent Difference $\pm 1.96 \times$

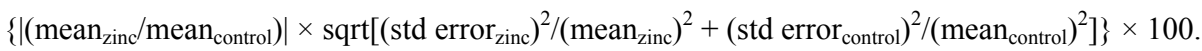

Table 5. Pooled relative risk of select outcomes for Chinese studies.

\begin{tabular}{|c|c|c|c|c|c|}
\hline \multirow[t]{2}{*}{ Outcome } & \multirow{2}{*}{$\begin{array}{c}\text { Specific } \\
\text { Causative } \\
\text { Pathogens }\end{array}$} & \multirow{2}{*}{$\begin{array}{c}\text { Study } \\
\text { Sites } \\
\quad N\end{array}$} & \multicolumn{2}{|c|}{$\begin{array}{c}\text { Pooled Estimate Percentage } \\
(95 \% \text { CI })^{2}\end{array}$} & \multirow{2}{*}{$\begin{array}{r}\text { Relative Risk }^{3} \\
\text { RR (95\% CI) }\end{array}$} \\
\hline & & & Zinc Group & Control Group & \\
\hline Episodes $>3$ & Unknown & 44 & $31.4(29.4-33.5)$ & $49.2(46.6-51.8)$ & $0.73(0.66-0.79)$ \\
\hline days $(\%)$ & Rotavirus & 29 & $31.8(29.5-34.1)$ & $50.3(47.4-53.3)$ & $0.70(0.63-0.78)$ \\
\hline $\begin{array}{c}\text { Episodes }>7 \\
\text { days }(\%)\end{array}$ & Unknown & 1 & $26.9(-)$ & $39.2(-)$ & $0.75(0.42-1.37)$ \\
\hline
\end{tabular}

We did not identify any studies reporting diarrhea-specific or all-cause mortality for inclusion in this review. Nor did we identify non-Chinese studies reporting duration of fever or vomiting, or Chinese studies reporting the proportion of children vomiting.

The mean episode duration and proportion of episodes lasting $>3$ days were not statistically significantly different comparing zinc-treated children in Chinese and non-Chinese studies. There was no statistically significant difference between the estimated relative risk of an episode lasting $>3$ days (RRR: 1.07; 95\% CI: 0.90-1.27) comparing Chinese and non-Chinese studies; therefore, we pooled this outcome across regions (RR: 0.74; 95\% CI: 0.68-0.80) (Figure 3). The percentage difference between the mean episode duration of zinc-treated and control group children was statistically significantly larger for Chinese compared to non-Chinese studies $(p<0.05)$, so this outcome was not pooled across regions. We did not have sufficient power to compare other commonly reported outcomes by region. 
Figure 2. Forest plot for the effect of therapeutic zinc supplementation on Rotavirus diarrhea episodes $>3$ days.

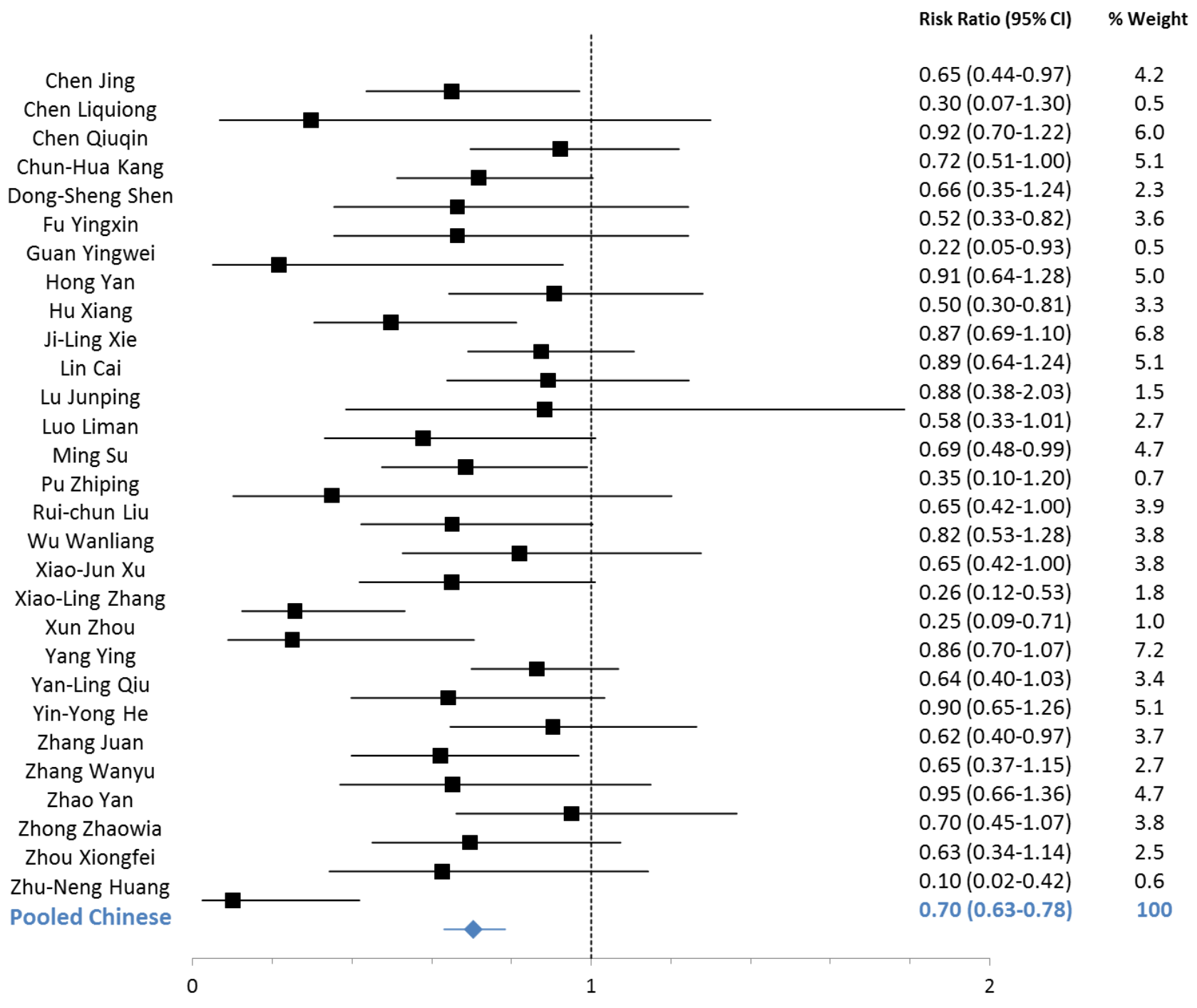

Zinc dose was not associated with the mean percent difference in diarrhea duration comparing zinc and control children for non-Chinese $(p=0.50)$ or Chinese $(p=0.12)$ studies. Comparing Chinese studies that used Licorzinc to those that used other zinc supplements, there were no statistically significant differences in the mean percent difference in the duration of rotavirus episodes $(p=0.56)$, the RR of non-specific episodes lasting $>3$ days (RRR: 0.99 ; 95\% CI: $0.72-1.35$ ), or the RR of rotavirus episodes lasting $>3$ days (RRR: 0.93; 95\% CI: 0.68-1.26). The percentage difference in the mean duration of non-specific episodes comparing zinc and control group children was statistically significantly higher for Licorzinc compared to "other zinc" studies $(p=0.01)$.

Our assessment of publication bias yielded largely symmetrical funnel plots for all outcomes.

Under the CHERG grading system, the studies included in this review were of moderate quality (Table 6) [11]. Effect estimates were largely consistent in directionality for all outcomes. 
Figure 3. Forest plot for the effect of therapeutic zinc supplementation on non-specific diarrhea episodes lasting $>3$ days.

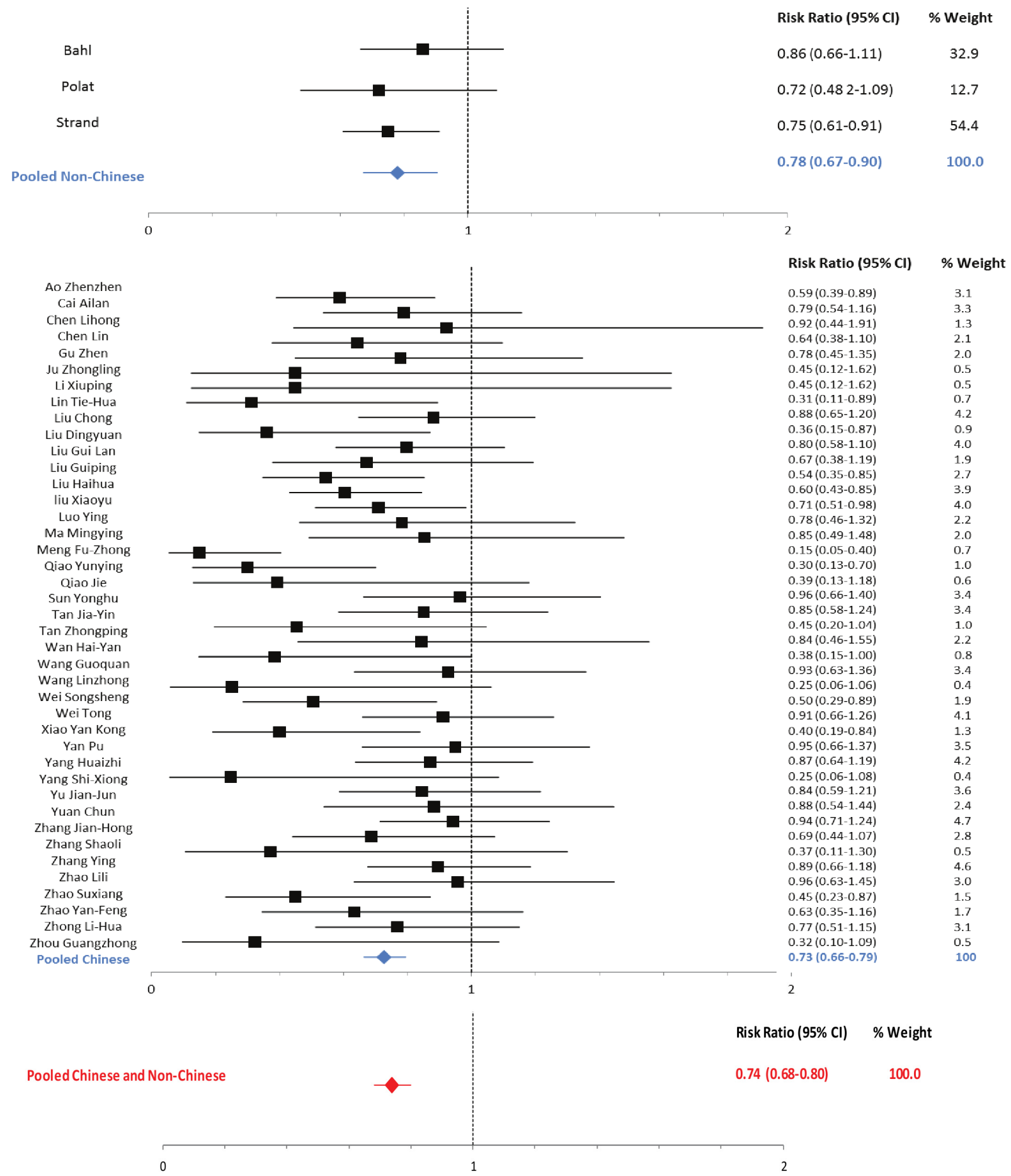


Table 6. Quality assessment of studies measuring the association between therapeutic zinc supplementation and selected outcomes.

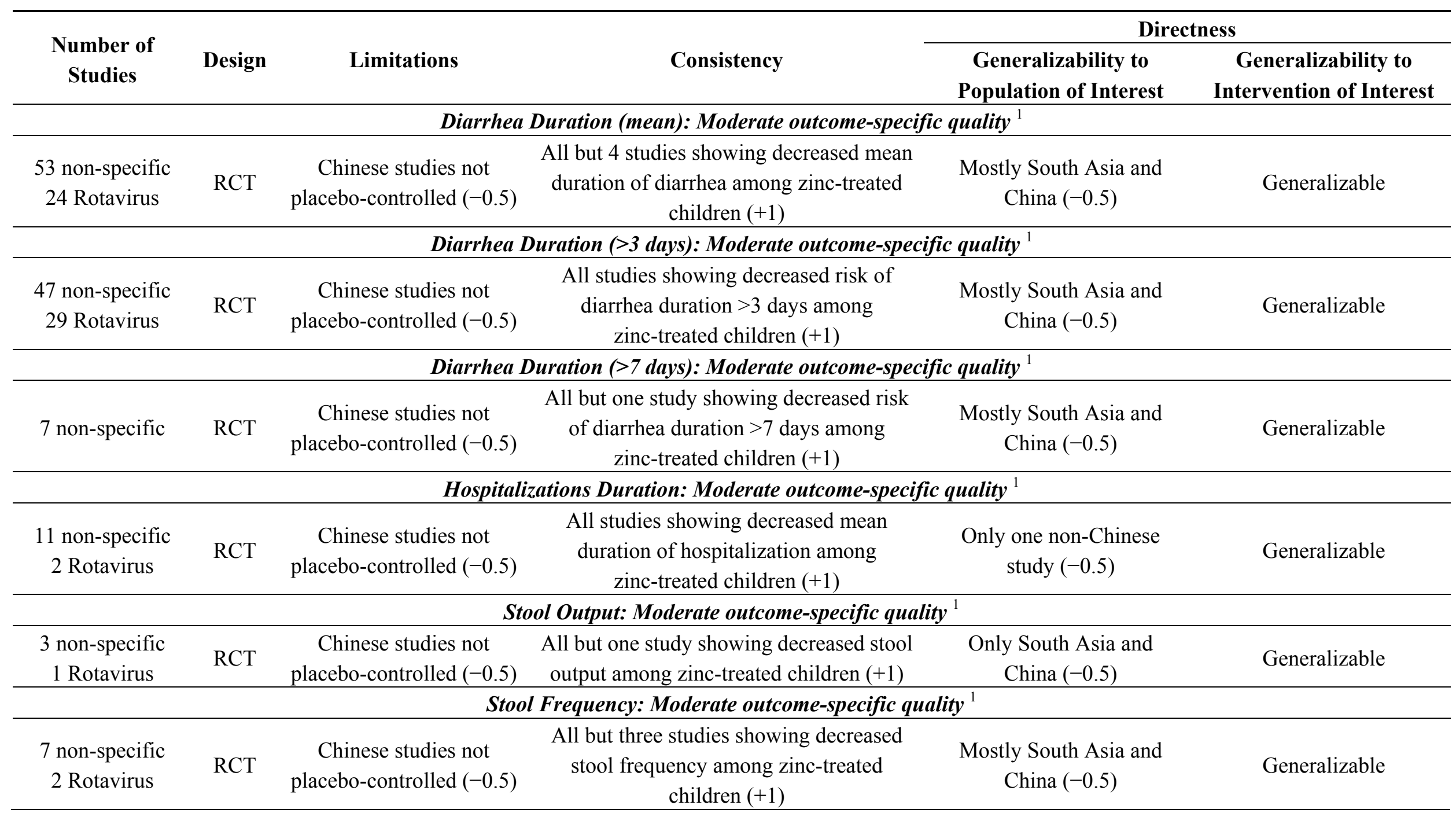


Table 6. Cont.

Vomiting: Moderate outcome-specific quality

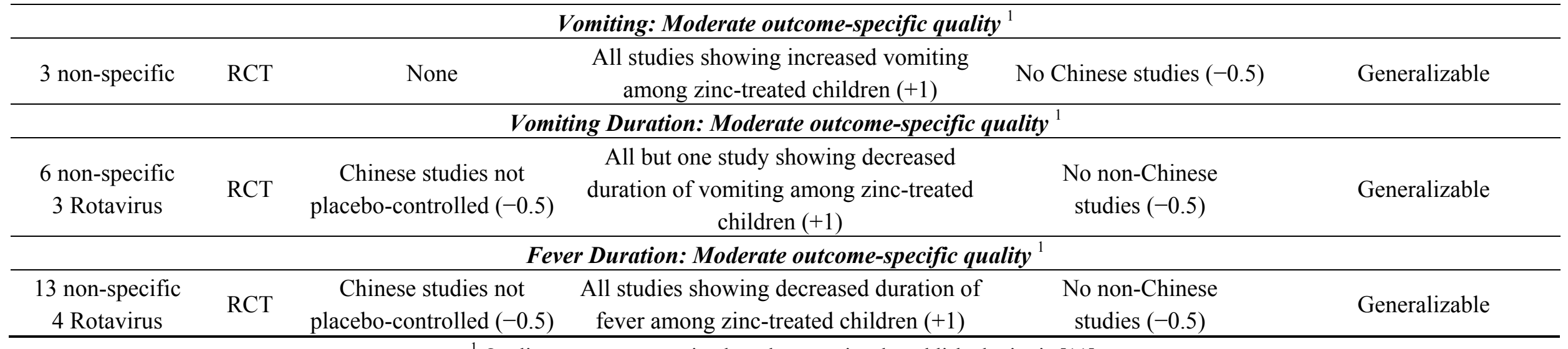

${ }^{1}$ Quality assessment scoring based on previously published criteria [11]. 


\section{Discussion}

The findings of our systematic review confirm and highlight the benefits of therapeutic zinc supplementation for diarrhea among children under five years of age in low- and middle-income countries. The effects of zinc treatment, which include reductions in episode duration, stool output, stool frequency and length of hospitalization, were consistent across Chinese and non-Chinese studies and non-specific and rotavirus diarrhea. These results suggest that zinc therapy of diarrhea is largely beneficial and important in both low- and middle-income settings.

The results of the large number of Chinese trials in rotavirus diarrhea are a substantial addition to the global evidence base because there have been no non-Chinese trials. One study in India based on a post-hoc subgroup analysis suggested that zinc treatment was not beneficial for rotavirus diarrhea [113]; however, the evidence from China demonstrates that therapeutic zinc supplementation reduces the duration and severity of rotavirus episodes. As rotavirus is the predominant cause of severe acute diarrhea worldwide and most likely the leading cause of diarrhea mortality [114], zinc treatment of rotavirus diarrhea could potentially yield large reductions in hospitalizations and deaths.

In comparison to non-Chinese studies, the difference between the mean episode duration of zinc-treated and control group children was statistically significantly higher for Chinese studies $(p<0.05)$. It is possible that this difference resulted from lack of placebo-controlled groups and blinding among Chinese studies. However, estimates of the effects of therapeutic zinc supplementation on other outcomes were largely consistent across study locations and we were able to generate a pooled global effect size for the proportion of episodes $>3$ days. The consistency of effect estimates between studies conducted in and outside China suggests that the lack of placebo-controlled groups in Chinese studies did not greatly bias the results.

Zinc dose did not affect the estimate of the effect of zinc supplementation on the duration of diarrhea for non-Chinese or Chinese studies. Although Licorzinc was associated with slightly greater reductions in the mean duration of non-specific diarrhea than other zinc products, zinc effect sizes were generally comparable across Chinese studies regardless of type of zinc preparation.

There is a dearth of literature meeting our inclusion criteria that assessed diarrhea-specific and all-cause mortality. Although a previous review published mortality effect estimates [4], the sole study reporting diarrhea-specific deaths was cluster-randomized and thus violated our inclusion criteria [115]. In addition, three studies of all-cause mortality were also excluded from our review; one was on persistent diarrhea [116], and two others were review papers [3,117].

Using previously published scoring criteria, the studies included in our review yielded pooled estimates of overall moderate quality [11]. The majority of studies contributing to this review were conducted in China and South Asia; however, studies conducted outside Asia were consistent in the directionality of effect estimates. The consistency and quality of all outcomes bolsters the evidence in support of oral zinc supplementation for the treatment of acute diarrhea among children under five in low- and middle-income countries. 


\section{Conclusions}

Oral therapeutic zinc supplementation reduces the morbidity of acute diarrhea among children under five in and outside China. Global efforts should be made to support scale-up of the WHO recommended regimen of therapeutic zinc in all regions.

\section{Authors' Contributions}

LML conducted the systematic review of non-Chinese studies, analysis and led the initial manuscript preparation. CLFW assisted with the analysis and the manuscript preparation. KC and WYJ conducted the systematic review of Chinese studies. REB conceptualized the idea and assisted with the interpretation of the analysis and the final manuscript preparation.

\section{Acknowledgments}

We would like to thank Wei-Ju Chen, Xun Luo and Wenze Zhang for reviewing and abstracting data from Chinese publications. Financial support for this review was provided by the Bill and Melinda Gates Foundation to the US Fund for UNICEF for the ongoing work of the Child Health Epidemiology Reference Group (CHERG).

\section{Conflicts of Interest}

The authors declare no conflict of interest.

\section{References}

1. Fischer-Walker, C.; Lamberti, L.; Roth, D.; Black, R. Zinc and Infectious Diseases. In Zinc in Human Health; Rink, L., Ed.; IOS Press: Amsterdam, The Netherlands, 2011; pp. 234-253.

2. WHO/UNICEF. Joint Statement: Clinical Management of Acute Diarrhoea; WHO/UNICEF: New York, NY, USA, 2004.

3. Lazzerini, M.; Ronfani, L. Oral zinc for treating diarrhoea in children. Cochrane Database Syst. Rev. 2013, 1, doi:10.1002/14651858.CD005436.

4. Fischer Walker, C.L.; Black, R.E. Zinc for the treatment of diarrhoea: Effect on diarrhoea morbidity, mortality and incidence of future episodes. Int. J. Epidemiol. 2010, 39, i63-i69.

5. Sachdev, H.P.; Mittal, N.K.; Mittal, S.K.; Yadav, H.S. A controlled trial on utility of oral zinc supplementation in acute dehydrating diarrhea in infants. J. Pediatr. Gastroenterol. Nutr. 1988, 7 , 877-881.

6. Sazawal, S.; Black, R.E.; Bhan, M.K.; Bhandari, N.; Sinha, A.; Jalla, S. Zinc supplementation in young children with acute diarrhea in India. N. Engl. J. Med. 1995, 333, 839-844.

7. Bahl, R.; Bhandari, N.; Saksena, M.; Strand, T.; Kumar, G.T.; Bhan, M.K.; Sommerfelt, H. Efficacy of zinc-fortified oral rehydration solution in 6- to 35-month-old children with acute diarrhea. J. Pediatr. 2002, 141, 677-682.

8. Zhang, J.S. Efficacy and effectiveness of 20 child health interventions in China: Systematic review of Chinese literature. J. Glob. Health 2011, 1, 87-95. 
9. Stata Statistical Software, release 12; StataCorp LP: College Station, TX, USA, 2011.

10. Altman, D.G.; Bland, J.M. Interaction revisited: The difference between two estimates. BMJ 2003, $326,219$.

11. Walker, N.; Fischer-Walker, C.; Bryce, J.; Bahl, R.; Cousens, S. Standards for CHERG reviews of intervention effects on child survival. Int. J. Epidemiol. 2010, 39, i21-i31.

12. Faruque, A.S.; Mahalanabis, D.; Haque, S.S.; Fuchs, G.J.; Habte, D. Double-blind, randomized, controlled trial of zinc or vitamin A supplementation in young children with acute diarrhoea. Acta Paediatr. 1999, 88, 154-160.

13. Roy, S.K.; Tomkins, A.M.; Haider, R.; Behren, R.H.; Akramuzzaman, S.M.; Mahalanabis, D.; Fuchs, G.J. Impact of zinc supplementation on subsequent growth and morbidity in Bangladeshi children with acute diarrhoea. Eur. J. Clin. Nutr. 1999, 53, 529-534.

14. Strand, T.A.; Chandyo, R.K.; Bahl, R.; Sharma, P.R.; Adhikari, R.K.; Bhandari, N.; Ulvik, R.J.; Molbak, K.; Bhan, M.K.; Sommerfelt, H. Effectiveness and efficacy of zinc for the treatment of acute diarrhea in young children. Pediatrics 2002, 109, 898-903.

15. Polat, T.B.; Uysalol, M.; Cetinkaya, F. Efficacy of zinc supplementation on the severity and duration of diarrhea in malnourished Turkish children. Pediatr. Int. Off. J. Jpn. Pediatr. Soc. 2003, 45, 555-559.

16. Brooks, W.A.; Santosham, M.; Roy, S.K.; Faruque, A.S.; Wahed, M.A.; Nahar, K.; Khan, A.I.; Khan, A.F.; Fuchs, G.J.; Black, R.E. Efficacy of zinc in young infants with acute watery diarrhea. Am. J. Clin. Nutr. 2005, 82, 605-610.

17. Al-Sonboli, N.; Gurgel, R.Q.; Shenkin, A.; Hart, C.A.; Cuevas, L.E. Zinc supplementation in Brazilian children with acute diarrhoea. Ann. Trop. Paediatr. 2003, 23, 3-8.

18. Larson, C.P.; Hoque, A.B.; Khan, A.M.; Saha, U.R. Initiation of zinc treatment for acute childhood diarrhoea and risk for vomiting or regurgitation: A randomized, double-blind, placebo-controlled trial. J. Health Popul. Nutr. 2005, 23, 311-319.

19. Fischer Walker, C.L.; Bhutta, Z.A.; Bhandari, N.; Teka, T.; Shahid, F.; Taneja, S.; Black, R.E. Zinc supplementation for the treatment of diarrhea in infants in Pakistan, India and Ethiopia. J. Pediatr. Gastroenterol. Nutr. 2006, 43, 357-363.

20. Patel, A.; Dibley, M.J.; Mamtani, M.; Badhoniya, N.; Kulkarni, H. Zinc and copper supplementation in acute diarrhea in children: A double-blind randomized controlled trial. BMC Med. 2009, 7, 22.

21. Elnemr, M.A.M.; Abdullah, A.K. Effect of zinc supplementation on morbidity due to acute diarrhoea in infants and children in Sanaa, Yemen: A randomized controlled double blind clinical trial. Sultan Qaboos Univ. Med. J. 2007, 7, 219-225.

22. Patro, B.; Szymanski, H.; Szajewska, H. Oral zinc for the treatment of acute gastroenteritis in Polish children: A randomized, double-blind, placebo-controlled trial. J. Pediatr. 2010, 157, 984-988. e1.

23. Dutta, P.; Mitra, U.; Dutta, S.; Naik, T.N.; Rajendran, K.; Chatterjee, M.K. Zinc, vitamin A, and micronutrient supplementation in children with diarrhea: A randomized controlled clinical trial of combination therapy versus monotherapy. J. Pediatr. 2011, 159, 633-637.

24. Zhao, L.; Li, X.; Sun, W. 40 Cases of oral licorzinc asist treatment of diarrhea. Chin. J. Esthet. Med. 2011, 20, 455 (in Chinese). 
25. Zhang, L. The effect of supplement zinc orally assist treatment on autumal diarrhea. Mod. Hosp. 2009, 9, 54-56 (in Chinese).

26. Lin, C.; Li, Q.; Zhang, M. Observation ofthe therapeutic efficiacy of oral zinc supplement with infantile rotaviral enteritis. Contemp. Med. 2010, 16, 8-10 (in Chinese).

27. Zhou, X.; Sun, Y.; Ouyang, J. Analysis on the therapeutic and preventative effects of zinc supplement on rotavirus enteritis in children. J. Xianning Univ. 2010, 24, 401-403 (in Chinese).

28. Yang, H. Clinical analysis of oral zinc adjuvant treatment for infants with acute diarrhea. China Mod. Med. 2011, 18, 7 (in Chinese).

29. Liu, G. Clinical observation on the therapeutic efficacy of oral zinc supplement in infants with acute diarrhea. J. Qiaihar Med. Coll. 2010, 31, 1416 (in Chinese).

30. Chen, L.; Bao, Y.; Gao, S. The comparison of the therapeutic effects on children diarrhea treated with smecta and oral zinc. J. Pediatr. Pharm. 2006, 12, 26-27 (in Chinese).

31. Liu, H.; Nie, X. Chinical observation on the therapeutic efficacy of oral zinc supplement in infants with diarrhea. Pract. Clin. Med. 2011, 12, 86-88 (in Chinese).

32. Liu, X.; Lan, X. Therapeutic effect of oral Zinc preparation on infantile diarrhea. Int. Med. Health Guid. News 2009, 15, 74-75 (in Chinese).

33. Fu, Y.; Zhang, W. Chinical analysis on the therapeutic efficacy of oral zinc supplement in infants with rotavirus enteritis. Guide China Med. 2010, 8, 260-262 (in Chinese).

34. Zhou, G. Clinical efficacy of licorzinc in treatment of infantile acute diarrhea. Youjiang Med. J. 2008, 36, 573-574 (in Chinese).

35. Chen, J. Clinical efficacy of licorzinc in treatment of infantile autumal diarrhea. J. Huaihai Med. 2008, 26, 442 (in Chinese).

36. Guan, Y.; Yingwei, Y.; Yu, J.; Zhou, Y. Clinical efficacy of licorzinc in treatment of infantile rotavirus enteritis. Stud. Trace Elem. Health 2012, 29, 21-22 (in Chinese).

37. Wu, W.; Li, F. 46 Cases of licorzinc in treatment of infantile rotavirus enteritis. Shaanxi J. Tradit. Chin. Med. 2010, 31, 296-297 (in Chinese).

38. Zhou, Y. Clinical observation of licorzinc treatment for children with diarrhea. Contemp. Med. 2010, 16, 20-21 (in Chinese).

39. Luo, L.E.A. Clinical observations on curative effects of licorzinc particles in adjuvant treating rotavirus enteritis. Clin. J. Med. Off. 2009, 37, 862-863 (in Chinese).

40. Zhang, S.; Wei, M. Observation of therapeutic effect of licorzinc granules adjuvant treatment for children with autumn diarrhea. Gems Health 2010, 5, 127-128 (in Chinese).

41. Ju, Z.; Zhang, Y.; Teng, L. Observation of therapeutic effect of licorzinc granules adjuvant treatment for children with autumn diarrhea. Shanxi Med. J. 2007, 36, 661 (in Chinese).

42. Wang, L. Observation of therapeutic effect of licorzinc granules treatment for 30 cases of children with autumn diarrhea. Chin. Pediatr. Integr. Tradit. West. Med. 2012, 4, 168-169 (in Chinese).

43. Hong, Y.; Wang, Z.; Shen, C. Clinical analysis of zinc sulfate adjuvant treatment for children with rotavirus enteritis. Zhejiang Clin. Med. J. 2009, 11, 845-846 (in Chinese).

44. Lin, G.; Wang, J.; Zhou, T. Observation of therapeutic effect of zinc sulfate treatment for 46 cases of autumn diarrhea. Stud. Trace Elem. Health 1994, 11, 61-62 (in Chinese).

45. Yan, M.; Liu, Y. Clinical analysis on efficacy of zinc sulfate in adjuvant treating 120 children with diarrhea. J. Taishan Med. Coll. 2011, 32, 303 (in Chinese). 
46. He, S.; Quan, Y.; Li, S. Determination of zinc in hair from infant patients with diarrhea and observation of curative effect with a supply of zinc. Guangdong Trace Elem. Sci. 1997, 4, 42-43 (in Chinese).

47. Wei, S. Observation of therapeutic effect of zinc gluconate treatment for children with acute diarrhea. Youjiang Med. J. 2011, 39, 50-52 (in Chinese).

48. Yang, C.; Xu, L.; Huang, Z. Observation of therapeutic effect of zinc gluconate adjuvant treatment for children with acute diarrhea. Chin. Community 2012, 14, 186 (in Chinese).

49. $\mathrm{Pu}, \mathrm{Z}$. Observation of therapeutic effect of zinc gluconate adjuvant treatment for children with autumn diarrhea. J. Clin. Exp. Med. 2010, 9, 1016-1017 (in Chinese).

50. Zhang, W. Analysis of therapeutic effect of zinc gluconate adjuvant treatment for children with rotavirus enteritis. China Mod. 2011, 49, 123-124 (in Chinese).

51. Sun, Y.; Sun, H.; Bian, X. Rotavirus enteritis suooprtive treated by glucoside zinc. J. Appl. Clin. Pediatr. 2008, 23, 1532-1533 (in Chinese).

52. Zhang, Y.; He, J. Observation of therapeutic effect of zinc gluconate adjuvant treatment for children with rotavirus enteritis. J. Med. Theory Pract. 2011, 24, 2319-2320 (in Chinese).

53. Lin, T.; Deng, J. Effect of zinc gluconate tablets on serum zinc and diarrhea of children with rotariras enteritis. J. Hainan Med. Univ. 2010, 16, 491-493 (in Chinese).

54. Liu, D. Clinical observation of zinc gluconate's effect on acute diarrhea of children. Sichuan Med. J. 2009, 30, 696-697 (in Chinese).

55. Qiao, J.; Wu, B. Observation of therapeutic effect of zinc gluconate treatment for children with acute diarrhea. Shanghai J. Prev. Med. 2011, 23, 581-582 (in Chinese).

56. Zhang, P.; Wu, J. Observation of therapeutic effect of zinc gluconate treatment for children with autumn diarrhea. Chin. Pract. J. Rural. 2007, 14, 29-30 (in Chinese).

57. Zhao, S.; Gao, Z. Observation of therapeutic effect of zinc gluconate treatment for children with autumn diarrhea. Chin. J. Clin. Res. 2012, 25, 463-464 (in Chinese).

58. Cai, A. The effect of zinc gluconate on infants with acutie diarrhea. Med. J. Qilu 2011, 26, 255-256 (in Chinese).

59. Zhang, J. Observation of curative effect of zinc gluconate in treatment of children rotavirus enteritis. Guide China Med. 2012, 10, 218-219 (in Chinese).

60. Qiao, R. Observation of therapeutic effect of zinc gluconate treatment for children with autumn diarrhea. Shanxi Med. J. 2012, 41, 380-381 (in Chinese).

61. Zhong, Z. Therapeutic effect of zinc gluconate on infantile rotavirus enteritis. Contemp. Med. 2012, 18, 130-131 (in Chinese).

62. Wang, Z. Observation of clinical efficacy of zinc gluconate on infantile rotavirus enteritis. China Med. Pharm. 2011, 1, 94-95 (in Chinese).

63. Ying, Y.; Mei, Q. Clinical observation of therapeutic effect of zinc gluconate on infantile rotavirus diarrhea. Chongqing Med. 2008, 37, 2442-2443 (in Chinese).

64. Zhao, Y. Clinical observation of therapeutic effect of zinc gluconate on infantile rotavirus diarrhea. China Foreign Med. Treat. 2012, 13, 113 (in Chinese).

65. Ma, Z. Validity and security of zinc gluconate on infantile rotavirus diarrhea. China J. Pharm. Econ. 2012, 2, 273-274 (in Chinese). 
66. Chen, Q.; Su, H. Efficacy and safety analysis of zinc gluconate in treatment of children with rotavirus diarrhea. Contemp. Med. 2012, 18, 144-145 (in Chinese).

67. Hu, X.; Zhang, X.; Chen, A. Observation of clinical efficacy of zinc gluconate on 60 cases with infantile acute duarrhea. Pract. Clin. Med. 2009, 10, 85 (in Chinese).

68. Yuan, C.; Guan, J. Therapeutic effect of zinc gluconate adjuvant treatment on infantile acute diarrhea. Pract. Clin. Med. 2011, 12, 76-77 (in Chinese).

69. Tan, Z. Observation of therapeutic effect of zinc gluconate adjuvant treatment for children with autumn diarrhea. Anhui Med. J. 2011, 32, 802-803 (in Chinese).

70. Liu, J. Clinical treatment and analysis in 69 Children with chronic diarrhea. Chin. Manip. Rehabil. Med. 2012, 3, 245-246 (in Chinese).

71. Hu, Y.; Deng, H. Observation of therapeutic effect of zinc gluconate granules for children with acute diarrhea. Chin. Foreign Women Health 2011, 19, 205 (in Chinese).

72. Li, X.; Wang, H.; Feng, G. Observation of therapeutic effect of zinc gluconate granules for children with autumn diarrhea. China Pharm. 2008, 11, 218-219 (in Chinese).

73. Gao, R. Clinical observation of supplemental zinc to treat 74 children with acute diarrhea. Asia Pac. Tradit. Med. 2012, 8, 58-59 (in Chinese).

74. Wu, H. The effect of zinc supplementation on children with diarrhea. Strait Pharm. J. 2011, 23, 156-158 (in Chinese).

75. Liu, J. Observation of therapeutic effects of zinc supplementation in treatment of children with acute diarrhea. Chin. J. Mod. Drug Appl. 2011, 5, 27-28 (in Chinese).

76. Chen, L.; Wang, P. Clinical analysis of zinc supplementation to treat infant diarrhea. Chin. J. Misdiagn. 2010, 10, 7582 (in Chinese).

77. Ma, K. Clinical observation of zinc supplementation to treat children with acute diarrhea. Chin. Foreign Med. Res. 2012, 10, 32-33 (in Chinese).

78. Lu, J. Clinical observation of zinc supplementation in treatment of autumn diarrhea. Med. Recapitul. 2012, 18, 1101-1102 (in Chinese).

79. Ma, M.; Yang, H. Clinical analysis of zinc preparation adjuvant treatment for children with acute diarrhea. Matern. Child Health Care China 2012, 27, 3847-3848 (in Chinese).

80. Ao, Z.; Wang, J.; Lin, L. Formulation adjuvant therapy efficacy in children with diarrhea. Hebei Med. 2012, 18, 1091-1093 (in Chinese).

81. Gu, Z.; Shen, H.; Zhao, P. Effect of zinc gluconate on acute diarrhea in children. J. Clin. Pediatr. 2011, 29, 249-251 (in Chinese).

82. Wen, Y.; Wang, W.; Yang, C. Zinc supplement in adjuvant treatment of children acute diarrhea. Chin. J. Prim. Med. Pharm. 2006, 13, 1208 (in Chinese).

83. Wang, G.; Wu, X. Observation of therapeutic effect of zinc adjuvant treatment for infantile autumn diarrhea. China Med. Pharm. 2011, 1, 66-98 (in Chinese).

84. Liu, R. Zinc therapy in the treatment of children with rotavirus enteritis. Jilin Med. J. 2012, 33, 5648 (in Chinese).

85. Liu, G. Observation of therapeutic effects of zinc supplementation in treatment of children with acute diarrhea. J. Med. Theory Pract. 2012, 25, 2287-2288 (in Chinese).

86. Tong, W. Observation of therapeutic effects of zinc supplementation in treatment of children with diarrhea. China Health Ind. 2011, 8, 84 (in Chinese). 
87. Qiu, Y. Serum zinc level of children with rotaviral diarrhea before and after zinc supplementation and clinical efficacy of the therapy. Chin. J. Woman Child Health Res. 2010, 21, 616-617 (in Chinese).

88. Kong, X. Cinical analysis of combination of lysine hydrochloride and zinc gluconate granules with ribavirin for children with diarrhea in autumn and winter. Inn. Mong. J. Tradit. Chin. Med. 2011, 30, 8 (in Chinese).

89. He, Y. Observation of therapeutic effects of oral zinc supplementation adjuvant treatment for 60 cases of rotavirus enteritis. Zhejiang J. Clin. Med. 2007, 9, 1635 (in Chinese).

90. Kang, C. Serum zinc levels and zinc supplementation treatment of young children with rotaviral enteritis. J. Kunming Med. Univ. 2010, 31, 109-113 (in Chinese).

91. Su, M. Observation of effect of serum zinc levels and zinc supplementation treatment of young children with rotaviral enteritis. China Health Ind. 2012, 6, 76-77 (in Chinese).

92. Huang, Z. Observation of therapeutic effects of zinc adjuvant treatment for 200 cases of infantile rotavirus enteritis. Chin. J. Ethnomed. Ethnopharm. 2010, 19, 80 (in Chinese).

93. Zhang, J. Observation of therapeutic effect of licorzinc granules with smectite powder for infantile diarrhea. Jiangxi Med. J. 2006, 41, 500-501 (in Chinese).

94. Wang, D. Observation of therapeutic effect of zinc treatment for infantile diarrhea. China Health Care Nutr. 2012, 5, 249 (in Chinese).

95. Lin, T. Observation of therapeutic effect of zinc gluconate adjuvant treatment for 60 cases of infantile acute diarrhea. Chin. Community 2008, 24, 29 (in Chinese).

96. Yan, P. Observation of therapeutic effect of zinc gluconate adjuvant treatment for children with acute diarrhea. China Foreign Med. Treat. 2011, 30, 118-119.

97. Yu, J. Clinical observation of therapeutic effect of zinc gluconate adjuvant treatment for 80 cases of children with diarrhea. Jilin Med. J. 2012, 26, 5644 (in Chinese).

98. Zhang, X. Effect of zinc gluconate among the treatment for infantile rotavirus diarrhea. Chin. J. Midiagn. 2011, 11, 7848 (in Chinese).

99. $\mathrm{Xu}, \mathrm{X}$. Observation of therapeutic effect of zinc gluconate treatment for children with rotavirus enteritis. Jiangsu Med. J. 2010, 36, 2327-2328 (in Chinese).

100. Tan, J. Observation of therapeutic effect of zinc gluconate treatment for children with autumn diarrhea. Med. J. Chin. People Health 2010, 22, 1122 (in Chinese).

101. Shen, D. Clinical observation of therapeutic Effect of zinc gluconate on infantile rotavirus enteritis. Guide China Med. 2012, 10, 46-47 (in Chinese).

102. Wang, J. Study of effect of zinc gluconate combined with Saccharomyces boulardii Sachets in treatment for children with autumn diarrhea. Contemp. Med. 2010, 16, 141-142 (in Chinese).

103. Chen, L. Clinical observation of therapeutic effect of Lysine and zinc gluconate adjuvant treatment for infantile acute diarrhea. Chin. J. Mod. Drug Appl. 2011, 5, 85-86 (in Chinese).

104. Meng, F. Observation of therapeutic effect of Lysine and zinc gluconate treatment for infantile autumn and winter diarrhea. Chin. Community 2012, 14, 152 (in Chinese).

105. Zhong, L. Observation of therapeutic effect of Lysine and zinc gluconateadjuvant treatment for infantile acute diarrhea. Chin. J. Aesthet. Med. 2010, 19, 258 (in Chinese).

106. Xie, J. Serum zinc level of rotaviral enteritis in children and the significance of zinc treatment. J. Pediatr. Pharm. 2010, 16, 18-20 (in Chinese). 
107. Fan, J. Application of zinc preparation in treatment for infantile diarrhea. Chin. J. Misdiagn. 2012, 12, 1306 (in Chinese).

108. Zhou, X. Therapeutic effect of zinc in the prevention and treatment of children rotvirus enteritis. Chin. Gen. Pract. 2012, 15, 1393-1394 (in Chinese).

109. Zhao, Y. Observation of effect of zinc preparation adjuvant treatment for infantile acute diarrhea. Zhejiang J. Prev. Med. 2008, 20, 44 (in Chinese).

110. Wan, H. Observation of therapeutic effect of Treasured zinc and selenium in adjuvant treatment for infantile diarrhea. Shandong Med. J. 2006, 46, 65 (in Chinese).

111. Yang, S. Observation of therapeutic effect of zinc preparation in adjuvant treatment for infantile autumn diarrhea. Health World 2012, 2, 244 (in Chinese).

112. Luo, Y. Observation of therapeutic effect of zinc adjuvant treatment for 168 cases of infantile acute diarrhea. Public Med. Forum Mag. 2012, 16, 3338 (in Chinese).

113. Patel, A.B.; Dibley, M.J.; Mamtani, M.; Badhoniya, N.; Kulkarni, H. Influence of zinc supplementation in acute diarrhea differs by the isolated organism. Int. J. Pediatr. 2010, 2010, doi:10.1155/2010/671587.

114. Fischer Walker, C.L.; Rudan, I.; Liu, L.; Nair, H.; Theodoratou, E.; Bhutta, Z.A.; O’Brien, K.L.; Campbell, H.; Black, R.E. Global burden of childhood pneumonia and diarrhoea. Lancet 2013, 381, 1405-1416.

115. Baqui, A.H.; Black, R.E.; El Arifeen, S.; Yunus, M.; Chakraborty, J.; Ahmed, S.; Vaughan, J.P. Effect of zinc supplementation started during diarrhoea on morbidity and mortality in Bangladeshi children: Community randomised trial. BMJ 2002, 325, 1059.

116. Roy, S.K.; Tomkins, A.M.; Mahalanabis, D.; Akramuzzaman, S.M.; Haider, R.; Behrens, R.H.; Fuchs, G. Impact of zinc supplementation on persistent diarrhoea in malnourished Bangladeshi children. Acta Paediatr. 1998, 87, 1235-1239.

117. Fontaine, O. Effect of zinc supplementation on clinical course of acute diarrhoea. J. Health Popul. Nutr. 2001, 19, 339-346.

(C) 2013 by the authors; licensee MDPI, Basel, Switzerland. This article is an open access article distributed under the terms and conditions of the Creative Commons Attribution license (http://creativecommons.org/licenses/by/3.0/). 